\title{
Du changement organisationnel au mouvement à partir d'une étude de cas dans l'université
}

Hugues Choplin, Jacques Audran, Eddie Soulier et Didier Paquelin

\section{(2) OpenEdition}

12 Journals

Édition électronique

URL : http://journals.openedition.org/communicationorganisation/458

DOI : 10.4000/communicationorganisation.458

ISSN : $1775-3546$

Éditeur

Presses universitaires de Bordeaux

Édition imprimée

Date de publication : 1 juin 2008

Pagination : 62-81

ISBN : 978-2-86781-506-5

ISSN : $1168-5549$

Référence électronique

Hugues Choplin, Jacques Audran, Eddie Soulier et Didier Paquelin, « Du changement organisationnel au mouvement à partir d'une étude de cas dans l'université », Communication et organisation [En ligne], 33 | 2008, mis en ligne le 01 juin 2011, consulté le 01 mai 2019. URL : http://journals.openedition.org/ communicationorganisation/458 ; DOI : 10.4000/communicationorganisation.458 
Dossier : Conduire le changement organisationnel?

\section{Résumé}

Cet article vise deux objectifs :

- sur un plan empirique : il s'agit d'analyser le déploiement du «e-learning » à des fins de changement organisationnel dans une université française. De ce point de vue, nous soulignons combien les normes de l'université étudiée en matière de collectifs et d'espaces-temps - compliquent ce changement ;

- sur un plan théorique : cette proposition vise à amorcer l'élaboration d'un paradigme, centré sur le mouvement - plutôt que sur le changement - des organisations. Sont ainsi proposées et illustrées trois catégories - celles d'événement, d'agencement et de potentiel - susceptibles de renouveler quelque peu nos modes d'analyse et d'action dans les organisations.

\section{Mots-clés}

e-learning, espaces-temps, événement, mouvement, université

\section{Abstract}

Our contribution relies on two objectives:

- on the empirical side: we analyse the deployment of an "e-learning" context as an organizational change in a French university. We thus underline how norms of the observed university - in terms of groups and time-space paradigm - make such change rather difficult;

- on a theoretical aspect, this proposal starts the construction of a paradigm, focused on "movement" - rather than change - of organizations including firms. The categories here described (events, agencement, potentiality) should slightly renovate analytical methods and actions in organizations.

Key- Words

e-learning, time-space, event, movement, university

\section{Hugues CHOPLIN}

Hugues Choplin est enseignant-chercheur en philosophie et directeur du département «Technologie et Sciences de l'Homme » à l'Université de Technologie de Compiègne et membre de l'unité COSTECH (EA, 2223).

\section{Jacques AUDRAN}

Jacques Audran est maître de conférences en sciences de l'éducation à l'Université de Provence - Aix-Marseille 1. Il est chercheur au sein de l'UMR ADEF (Apprentissage Didactique Evaluation et Formation).

\section{Eddie SOULIER}

Eddie Soulier est enseignant-chercheur en système d'information et gestion à l'Université de Technologie de Troyes, directeur du Centre d'Innovation Pédagogique de l'UTT et membre du Laboratoire Tech-CICO FRE CNRS 2848.

\section{Didier PAQUELIN}

Didier Paquelin est maître de conférences en sciences de l'information et de la communication à l'Université Michel de Montaigne - Bordeaux 3, et chercheur à IMAGINES, Images, Histoire et Sociétés, dans cette Université. 


\title{
Du changement organisationnel au mouvement à partir d'une étude de cas dans l'université
}

\author{
Hugues Choplin, Jacques Audran, \\ Eddie Soulier, Didier Paquelin
}

hugues.choplin@utc.fr, jacques.audran@univ-provence.fr, eddie.soulier@utt.fr, paquelin@u-bordeaux3.fr

Cet article vise deux objectifs :

sur un plan empirique: il s'agit d'analyser le déploiement du «e-learning » à des fins de changement organisationnel dans une université française ;

sur un plan théorique : cette proposition vise à amorcer l'élaboration d'un paradigme spécifique, centré sur le mouvement - plutôt que sur le changement - des organisations (entreprises comprises).

\section{Un partenariat entre l'UTT et une ERTé}

L'Université de Technologie de Troyes (UTT) s'attache depuis 2002 à déployer des outils de e-learning dans son enseignement via un dispositif spécifique: TEMIS (Technologies Educatives pour le Management de l'Information et des Savoirs). Le Centre d'Innovation Pédagogique (CIP), porteur de cette mission, cherche non seulement à développer de nouvelles pratiques pédagogiques mais aussi à « impacter » l'organisation même de l'enseignement dans l'Université de telle façon qu'elle puisse se centrer davantage sur les activités d'apprentissage des étudiants. Tranchant sur celles pédagogiques ou technologiques, des autres centres français du même type, la démarche spécifique retenue dans cette perspective se veut d'une part déterminée par une orientation «client», mettant au cœur de ses préoccupations les services aux étudiants et aux enseignantschercheurs, et d'autre part inspirée par des approches théoriques de l'innovation en termes de réseaux socio-techniques (Latour, 2006). 


\section{Dossier : Conduire le changement organisationnel?}

C'est dans ce contexte que s'est établi un partenariat entre le CIP et une Equipe de Recherche en Technologie de l'éducation (ERTé) centré sur le thème: "Campus numérique et innovation pédagogique ${ }^{1}$. L'innovation pédagogique désigne ici non pas seulement de nouveaux objets - telles les technologies de l'information et de la communication (TIC) - mais aussi des processus complexes s'appuyant sur ces nouveaux objets. Flous, difficilement prévisibles, ces processus socio-techniques donnent lieu à une transformation des conceptions et des pratiques, des modes de relation entre acteurs et, davantage encore, de l'organisation, des valeurs et des finalités d'une institution (Cros, 1998). En ce sens, l'innovation pédagogique se rapproche de l'innovation au sens d'Alter (2000) ou du changement organisationnel tel qu'il est visé par les acteurs du CIP. Le partenariat établi vise également à la formulation de scénarii concrets de (re)déploiement de TEMIS et de l'action du CIP.

\section{Eléments de méthode}

Relevant d'une recherche pour l'action (plutôt que d'une rechercheaction), notre approche s'attache d'une part à conjuguer différentes disciplines scientifiques (principalement: philosophie, sciences de l'information et de la communication, sciences de l'éducation et sociologie) et d'autre part à articuler recherche scientifique et pratiques opérationnelles.

Notre travail a d'abord procédé d'une enquête empirique, empruntant aux méthodes de l'ethnosociologie :

notes de terrain, observations directes à l'occasion de cinq visites d'une journée ou de deux jours à l'UTT ;

analyses d'artefacts informatiques (sites web, écrans, outils), des forums étudiants et des weblogs de l'UTT ;

vingt entretiens multi-acteurs (CIP, enseignants, étudiants, direction) enregistrés et retranscrits.

Cette enquête empirique a été doublement régulée :

par des échanges et des dissensus avec les acteurs de terrain du CIP (régulation «interne ») concernant les constats empiriques effectués ou la stratégie d'action du CIP ;

\footnotetext{
${ }^{1}$ Cette équipe, labellisée par le Ministère de l'Enseignement Supérieur et de la Recherche (ERTé $\left.n^{\circ} 59\right)$, est portée par l'université de Provence, l'université de Bordeaux 3 et l'Université de Technologie de Compiègne.
} 
au sein de l'équipe ERTé, que ce soit au niveau des autres terrains (ou campus numériques) étudiés en parallèle dans l'équipe ou au niveau du projet théorique global de l'ERTé (Paquelin et al., 2006) (régulation « externe »).

\section{Un changement organisationnel qui ne « prend » pas}

L'enquête empirique conduite montre que TEMIS ne remplit pas aujourd'hui la fonction visée de changement. Certes, le dispositif est déployé de manière très significative dans l'UTT - y compris sur le plan des usages, variés et généralisés ${ }^{2}-$, au point qu'il semble constituer une référence au niveau national. Mais, en dépit de la démarche spécifique qui le sous-tend, ce déploiement ne paraît pas impliquer un véritable processus de changement organisationnel : TEMIS ne fait qu'effleurer les pratiques existantes sans les modifier en profondeur.

$\mathrm{Ce}$ point est particulièrement net au niveau des étudiants. Globalement, de leur point de vue, l'UTT répond à leurs attentes en matière d'accueil et d'équipement: "c'est une université récente, neuve, avec de bons moyens informatiques » nous dit un étudiant du cycle «ingénieur $»^{3}$. Même s'ils ont conscience du potentiel de cette université de technologie en matière de ressources numériques, les étudiants insistent davantage sur les relations «plus intéressantes avec les professeurs», la proximité de ces derniers, la présence de «beaucoup de pratique » et de «concret» dans les études, et ce en contraste avec le lycée. Dans ce paysage, TEMIS semble leur paraître bien abstrait - n'est-ce pas seulement nos interviews qui leur rendent le dispositif visible (Latour, 2006)? Lorsque les fonctions du dispositif sont évoquées, c'est de manière pragmatique : «c'est ici qu'on va voir nos notes, qu'on dépose nos rapports de projet, qu'on cherche (...) les exos et les corrigés ».

Plus encore : alors que nous les questionnons sur TEMIS, les étudiants nous parlent d'un autre site Internet : « siteétu». Depuis 2000, ce site alternatif - conçu et développé par des étudiants de l'UTT (mais hébergé sur les machines de l'université) - «a explosé » en termes d'usage. Il y est question d'abord de la vie étudiante (vie à Troyes, co-voiturage) mais aussi d'aspects pédagogiques : trombinoscope des

\footnotetext{
${ }^{2}$ Mentionnons ici qu'au début 2007 la totalité des étudiants et la moitié des enseignants étaient concernés par le dépôt ou l'accès aux ressources pédagogiques en ligne.

${ }^{3}$ Le cursus à l'UTT (de 5 ans) est divisé en deux cycles: le cycle du «tronc commun » (deux premières années) et le cycle « ingénieur » (trois dernières années).
} 
Dossier : Conduire le changement organisationnel?

enseignants; commentaires libres sur les Unités de Valeur par les étudiants ; informations sur les cours annulés/déplacés ; échanges à distance entre les étudiants en stage...

De ce point de vue, nos entretiens avec les étudiants révèlent plus des pratiques invisibles - non prescrites par l'institution - qu'ils n'attestent de transformation de pratiques. On n'en conclura pas que TEMIS n'est pas utilisé : il l'est à la manière d'une distribution de services « ordinaires » de l'université.

Un constat comparable semble pouvoir être effectué au niveau des enseignants-chercheurs : tout se passe comme si TEMIS, tout en étant utilisé, passait «à côté » du noyau des activités des acteurs ${ }^{4}$, comme si ceux-ci ne faisaient qu'y piocher des éléments locaux en fonction de leurs besoins immédiats, chaque acteur ayant finalement une vision très parcellaire de TEMIS. Dans ces conditions, TEMIS ne saurait remettre en cause les pratiques existantes. Il ne saurait non plus constituer, au sein de l'UTT, un médiateur effectif ou un authentique actant (Latour, 2006) ${ }^{5}$.

Comment rendre compte de ces éléments empiriques, de cet émiettement des pratiques et de cette absence d'innovation pédagogique et de changement organisationnel ? La question nous semble d'autant plus aiguë que le CIP s'attache très significativement à «impacter» l'organisation de l'UTT, conduit qu'il est par un acteur à la fois sociologue et consultant, spécialiste des dynamiques organisationnelles et socio-techniques. Deux hypothèses - non exclusives l'une de l'autre - semblent pouvoir être émises touchant cette question ${ }^{6}$.

\section{Hypothèse 1 : les normes de l'UTT freinent les possibilités du changement}

On pourrait de prime abord penser que la démarche retenue par le CIP est inadaptée à l'univers éducatif et à ses traits culturels et organisationnels. L'orientation «client » n'est-elle pas d'abord une pratique issue de l'univers de l'entreprise ? De surcroît, dans quelle

\footnotetext{
${ }^{4}$ Nos entretiens semblent confirmer le poids des exigences spécifiques de la recherche dans les représentations et les pratiques des enseignants-chercheurs.

${ }^{5}$ Il faudrait expliciter ici les liens entre cette médiation et la transformation de pratiques.

${ }^{6}$ A la lumière de l'enquête empirique conduite, nous estimons ne pas disposer d'éléments suffisants pour analyser et comparer la pertinence relative de ces deux hypothèses (étude qui exigerait un autre travail spécifique, lequel conduirait en particulier à approfondir les dimensions mobilisées dans l'hypothèse 2).
} 
mesure les réseaux socio-techniques ont-ils déjà été éprouvés dans l'univers éducatif ? Il nous paraît toutefois inexact de rendre compte de la situation à l'UTT en questionnant d'abord la posture et la démarche du CIP. Ce serait en effet négliger combien la majorité des enquêtes effectuées depuis maintenant plusieurs années sur les dispositifs de type «campus numérique » montrent que l'introduction de ces dispositifs ne modifient pas substantiellement les pratiques pédagogiques des enseignants (de la conception à la mise en œuvre de l'acte pédagogique) et les processus d'apprentissage (Paquelin et al., 2006). De ce point de vue, l'importance de la démarche retenue par leurs porteurs - aussi spécifique soit-elle - semble bien devoir être relativisée. Comme si, quelle que soit la nature de cette démarche, ces dispositifs venaient se heurter au cadre même régissant la pratique de l'enseignement.

Dès lors, notre première hypothèse est de soutenir que si la mise en œuvre de TEMIS ne suscite pas de changement, c'est parce qu'elle n'est pas en mesure de donner lieu à une transformation des normes et des règles de l'action pédagogique qui prévalent dans une université comme l'UTT. Les entretiens effectués confèrent une certaine pertinence à cette hypothèse. Plus précisément, ils conduisent à comprendre ces normes et ces règles selon deux dimensions dont on sait que la dynamique est déterminante dans la mise en mouvement de ces bureaucraties professionnelles (selon la formule de Mintzberg) que constituent les universités : les collectifs et les espaces-temps ${ }^{7}$.

Les collectifs pédagogiques, tout d'abord, semblent sinon inexistants du moins très limités dans l'université. Sans doute quelques collectifs disciplinaires existent-ils, mais de façon générale il en est très peu question dans les entretiens. L'atteste par exemple la nécessité aujourd'hui de créer une équipe d'enseignants pour animer et réguler le cycle du «tronc commun». Une actrice du CIP se dit particulièrement surprise de cette absence d' équipe pédagogique (...) c'est une des choses qui m'a frappée quand je suis arrivée ici. » Le responsable du CIP, également enseignant-chercheur à l'UTT, dit: "j'ai vraiment vécu l'absence totale de réunions pédagogiques ». Mais comment donc interroger les pratiques pédagogiques en vigueur sans constitution de collectifs adhoc (Bonami et Garant, 96 ; Cros, 98) ?

\footnotetext{
${ }^{7}$ C'est peut-être la spécificité du projet théorique de notre ERTé que de se centrer sur des dimensions - les collectifs, les espaces-temps et également les objets intermédiaires - qui conduisent à mettre quelque peu au second plan ces acteurs individuels que sont l'étudiant et l'enseignant.
} 
Dossier : Conduire le changement organisationnel?

Touchant la dimension des espaces-temps pédagogiques, il apparaît que la logique dominante «cours / TD / TP», qui plus est dans un seul site géographique (à Troyes), limite fortement la possibilité de transformations pédagogiques significatives. Plusieurs témoignages l'attestent, par exemple celui d'un enseignant-chercheur, également directeur à la pédagogie: [à propos de l'usage pédagogique des forums électroniques] "c'est moi qui n'ai pas toujours la réactivité donc je ne fais pas vraiment forum, je préfère répondre directement en cours (...) donc là je travaille plutôt en direct avec les étudiants»; «en termes d'intégration dans le processus pédagogique, ça reste quand même relativement léger (...) c'est plutôt de l'aide au cours, aux travaux dirigés, un accompagnement $»$. Ces éléments suggèrent que le poids des espaces-temps classiques de l'enseignement et des cours «condamne » le dispositif TEMIS à accompagner ou à enrichir - plutôt qu'à transformer - le processus pédagogique traditionnel.

Ainsi appréhendée, notre première hypothèse se formule donc en ces termes : c'est parce que la mise en œuvre du dispositif TEMIS ne donne lieu ni à la remise en cause de l'absence de collectifs pédagogiques ni à la reconfiguration des espaces-temps de l'enseignement traditionnel qu'elle ne peut susciter le changement visé.

\section{Hypothèse 2 : la complexité du mouvement à l'UTT rend difficile l'action du CIP}

Faut-il dès lors soutenir que la démarche du CIP est vouée à l'échec et que toute visée de changement organisationnel doit être abandonnée ? Sans doute pas. Il nous semble en effet que cette première analyse mettant en évidence la force et la stabilité des normes pédagogiques de l'UTT - n'est pas suffisante, ne serait-ce que parce qu'elle paraît trop caricaturale pour une université jeune (créée en 1993) qui demeure encore «à construire » (dixit un enseignant-chercheur). Il est possible que sur le plan pédagogique, la configuration de l'UTT soit relativement stable aujourd'hui. Toutefois, il paraît difficile de soutenir que ce constat rend compte de la complexité actuelle de l'UTT et du potentiel d'action qui s'offre peut-être au CIP, y compris à terme sur le plan pédagogique. Telle est en effet notre seconde hypothèse : c'est parce que le CIP n'a pas pris la mesure de cette complexité qu'il ne parvient pas à susciter le changement organisationnel visé. Mais comment décrire cette complexité ?

Visant à renouveler dans une certaine mesure nos approches théoriques des dynamiques organisationnelles, notre proposition est 
d'aborder cette complexité de l'université en termes de mouvement. De notre point de vue, l'UTT est en effet traversée par un mouvement probablement irréductible aux processus de changement décrits «traditionnellement » par les théoriciens et praticiens du changement organisationnel, lesquels restent, nous semble-t-il, le plus souvent déterminés par une pensée du changement (ou du processus) comme apprentissage (individuel ou/et organisationnel) (par exemple Bernoux, 2004). Cette approche nous semble légitimée par le constat de désordre (Alter, 2000) ou d'émiettement des activités de l'UTT suggéré par nos analyses de terrain touchant les usages de TEMIS. N'est-il pas a priori réducteur de rabattre ce désordre ou cet émiettement sur ces processus particuliers que sont les processus d'apprentissage ? Nous proposons dès lors d'interroger l'UTT à partir des trois dimensions suivantes constitutives du mouvement en tant qu'il déborde (partiellement au moins) ces processus :

l'évènement (Badiou, 1988): dans quelle mesure l'innovation managériale (Segrestin, 2004) initiée en 2005 par la mise en place d'une organisation matricielle dans l'université constitue-t-elle un événement dont les conséquences sont susceptibles de transformer en profondeur l'institution? Qui sont les sujets - individuels ou collectifs - qui tirent les conséquences (éventuelles) de cet événement ? Il semblerait qu'une incertitude règne à l'UTT quant à ces questions, liées à l'arrivée, au sein de la direction, de managers issus du privé et peu au fait de la culture des enseignants-chercheurs. «Avec la nouvelle organisation je m'interroge, et les collègues aussi, sur l'avenir de notre métier » nous dit un enseignant-chercheur ;

l'agencement (Deleuze et Parnet, 1977) ${ }^{8}$ : dans quelle mesure l'UTT agence-t-elle ou conjugue-t-elle des mondes hétérogènes (mondes de la recherche, de la rationalisation, du service...) ? Il semblerait que la dynamique actuelle de l'UTT procède moins d'un tel agencement que de l'addition - dont on peut penser qu'elle est globalement désordonnée - de dynamiques propres et internes à ces mondes. Nous l'avons suggéré en évoquant l'émiettement lié à TEMIS. Nos entretiens semblent également marquer l'écart entre le monde des enseignants-chercheurs et celui des managers: «de plus en plus de chefs, mais le cour de métier n'est plus là» nous explique ainsi un enseignant-chercheur à propos de ces derniers ;

\footnotetext{
${ }^{8} \mathrm{Au}$ sens de Deleuze, l'agencement engage deux caractéristiques que nous reprenons ici : a) il conjugue des dimensions hétérogènes (qu'il agence); b) il procède d'une dynamique ou d'un devenir spécifique.
} 
Dossier : Conduire le changement organisationnel?

les potentiels (Simondon, 1989) propres à l'UTT : se distinguant à la fois de simples circonstances et de possibles (Bergson, 1907), ces potentiels désignent des propensions (Jullien, 1996) ou encore des devenirs significatifs à l'œuvre au sein et/ou à l'extérieur de l'institution. Il ne semble pas qu'aujourd'hui TEMIS relève d'une telle accumulation de potentiels : le dispositif désigne plutôt des possibles engageant une offre de services - qu'il importerait de «brancher» sur d'authentiques potentiels. Lesquels ? Deux potentialisations émergent semble-t-il dans la situation actuelle de l'UTT :

l'autonomisation de l'université (au regard des tutelles ministérielles) qui semble faire système avec la mise en œuvre de procédures de rationalisation issues du privé. Cette autonomisation pourrait-elle conduire l'UTT - et les différents mondes qui la constituent - à établir une stratégie d'enseignement susceptible d'intégrer un dispositif comme TEMIS ?

la pression due à un recrutement après le bac (d'étudiants en science) de plus en plus difficile. TEMIS ne peut-il constituer de ce point de vue un facteur d'innovation pédagogique significatif et attractif pour des publics plus diversifiés qu'ils ne le sont aujourd'hui ?

\section{Perspectives opérationnelles et théoriques}

Autonomisation, pression du recrutement : ces deux points semblent engager une forme de situation de péril (Jullien, 1996) pour l'UTT, propice sans doute au mouvement et aux transformations en profondeur de l'université. N'est-ce pas dès lors en priorité à l'aune de ces deux potentialisations que doit aujourd'hui essayer de se reconfigurer la démarche du CIP ?

Evénement (vs objectif), agencement (vs organisation), potentiel (vs projet et processus) : l'intégration de ces trois catégories complémentaires paraît bien ainsi donner prise à une nouvelle lecture des possibilités d'action dans une institution comme l'UTT. Sans doute s'agit-il dès lors moins de conduire le changement organisationnel que d'agir dans le mouvement (Choplin et al., 2007). C'est précisément pour définir ces modes spécifiques d'action dans le mouvement qu'ont été construits et lancés le programme et le réseau de Recherche finalisée dans les Entreprises et les Organisations $(\text { REMEO })^{9}$.

${ }^{9}$ Dont les porteurs sont l'UTC, l'UTT et l'ESTIA. 
Du changement organisationnel au mouvement

\section{Bibliographie}

ALTER, Norbert. L'innovation ordinaire. Paris : PUF, 2000, 278 p.

BADIOU, Alain. L'être et l'événement. Paris : Seuil, 1988, 566 p.

BERGSON, Henri. L'évolution créatrice. Paris : PUF, 1907, 384 p.

BERNOUX, Philippe. Sociologie du changement dans les entreprises et les organisations. Paris : Seuil, 2004, 308 p.

BONAMI, Michel., GARANT, Michèle., eds. Systèmes scolaires et pilotage de l'innovation. Emergence et implantation du changement. Paris, Bruxelles : De Boeck, 1996, 238 p.

CHOPLIN, Hugues, SOULIER, Etienne, BACHIMONT, Bruno. Eléments pour une recherche finalisée sur le mouvement. Cognitique, Cognitics, Série de Sciences Cognitives Appliquées, n¹1, 2007, p. 99113.

CROS, Françoise, ed. Dynamiques du changement en éducation et en formation. Versailles : INRP/IUFM, 1998, $284 \mathrm{p}$.

DELEUZE, Gilles, PARNET, Claire. Dialogues. Paris : Flammarion, 1977, $187 \mathrm{p}$.

JULLIEN, François. Traité de l'efficacité. Paris : Grasset, 1996, 234 p.

LATOUR, Bruno. Changer la société Refaire de la sociologie. Paris : La Découverte, 2006, 400 p.

PAQUELIN, Didier, AUDRAN, Jacques, CHOPLIN, Hugues, HRYSHCHUK, Svitlana, SIMONIAN, Stéphane. Campus numérique et innovation pédagogique: l'hypothèse de la territorialisation. Distances et savoirs, vol. 4, n³, 2006, p. 365-395.

SEGRESTIN, Denis. Les chantiers du manager. Paris: A. Colin, 2004, 343 p.

SIMONDON, Gilbert. L'individuation psychique et collective. Paris : Aubier, 1989, 293 p. 
Dossier : Conduire le changement organisationnel?

\title{
Résumé
}

A ce jour, peu de recherches ont étudié les effets du management de la qualité sur les rationalités narrative et argumentative. Cette étude a pour objectif de montrer que la mise en œuvre collective de la démarche qualité favorise le développement d'une structure narrative. Inversement, l'introduction des principes de la qualité selon le style managérial «top-down» génère un phénomène de résistance au changement et une structure argumentative.

\section{Mots-clés}

Changement organisationnel, management de la qualité, référentiel, implication, établissement médico-social

\begin{abstract}
To date little research has examined the effects of the quality management on the narrative rationality and the argumentative rationality. The purpose of this study is to show that the collective quality management implementation contributes to the development of narration. Conversely, the implementation of the quality principles in accordance to the top-down management style generates a "resistance to change" phenomenon and argumentative rationality.
\end{abstract}

\section{Key-Words}

Organizational change, quality management, systeme of reference, involvement, health centre

\section{Youcef AÏSSANI}

Youcef Aïssani est maître de conférences à l'Université Paul Sabatier - Toulouse III (IUT Tarbes). Il y enseigne la communication et la psychosociologie. Il est co-responsable de l'équipe Changements Organisationnels et Qualité au LERASS.

\section{Odile BORDES}

Odile Bordes est maître de conférences à l'Université Paul Sabatier Toulouse III (IUT A). Elle y enseigne la communication, la qualité des services et la psychosociologie. Elle est membre du Lerass; ses travaux portent sur les effets organisationnels de la démarche qualité. 


\section{De l'organisation narrative à l'organisation argumentative : les effets paradoxaux d'un référentiel qualité}

Youcef Aïssani, Odile Bordes

youcef.aissani@iut-tlse3.fr

odile.bordes@iut-tlse3.fr

Selon Weick, les organisations peuvent se répartir en deux grandes catégories selon qu'elles procèdent d'une logique narrative ou argumentative. Cette distinction permet de classer les organisations sur la base de critères de communication. Aujourd'hui largement admise, elle soulève la question, à ce jour relativement peu explorée, du passage d'une forme à l'autre. Dans le cadre de cette étude, nous nous emploierons à cerner le déterminant et les modalités du changement organisationnel. Après avoir rappelé la définition de ces types d'organisation, nous montrerons l'intérêt d'aborder la question en se plaçant au croisement de deux perspectives : le paradigme de l'instrument de gestion et l'approche pragmatique de Weick. Enfin, nous présenterons les résultats de notre investigation empirique centrée sur les effets paradoxaux d'un outil de gestion en vogue : le référentiel qualité.

\section{Organisations narrative et argumentative}

La mouvance interactionniste dans laquelle l'œuvre de Weick s'inscrit a conduit l'auteur à déployer une réflexion critique des organisations. Contre l'approche holiste et réifiante de l'organisation, longtemps dominante, Weick développe une perspective centrée sur les microprocessus communicationnels qui font la vie organisationnelle. Il considère que l'organisation se confond avec l'ensemble des interactions réciproques. La communication ne se ramène donc nullement à un sous-système de l'organisation, comparable aux autres sous-systèmes que sont la production, la finance, les ressources humaines, et pas davantage à un dispositif de gestion destiné à l'amélioration de la performance globale. Elle est fondatrice de l'ordre organisationnel (Giordano, 2006). Considérant ainsi que l'architecture globale d'une organisation découle des caractéristiques des 
communications qu'y tissent les acteurs, Weick et Browning (1986) proposent de distinguer deux formes majeures d'organisation. Dans l'organisation argumentative, le discours de la rationalité et l'instrumentation de gestion sont érigés en principes organisateurs de l'action collective. L'organisation est appréhendée comme un tout homogène, constitué d'activités articulées entre elles selon des dispositifs et des règles rationnels. Dominée par une logique formelle et fonctionnelle (description des procédures à suivre, élaboration d'instruments de contrôle, rappel des objectifs à atteindre, discours de légitimation...), la communication se déploie sur un mode centralisé intégré à la hiérarchie. Dans l'organisation narrative d'autre part, l'accent est mis davantage sur les interactions interpersonnelles et les échanges entre services médiatisés par les acteurs. On a affaire, ici, à une communication riche en «histoires » (Browning) et en narrations, qui permet l'expression des multiples expériences individuelles ou groupales où s'entremêlent des contenus affectifs, imaginaires et des références au réel. Contrairement au discours argumentatif, les narrations opèrent sur un mode décentralisé appelant dialogue et participation. Ce type d'organisation favorise l'émergence de spécificités locales (normes et attitudes de groupe, communication ritualisée, formation de cultures de service...) et le développement de logiques d'action différenciées.

\section{Instrumentation de gestion et changement organisationnel}

A nos yeux, la valeur heuristique de cette distinction (qui n'est pas sans rappeler l'opposition entre les organisations mécaniques et organiques proposée par Burns et Stalker, 1961) ne se réduit pas à sa vertu taxonomique. Nous pensons en effet qu'elle peut s'étendre à la problématique du changement. Précisément, la typologie de Weick et Browning nous paraît contenir en germe la question du passage d'une forme organisationnelle à l'autre. Une organisation narrative peut-elle évoluer vers une forme argumentative (et vice versa) ? Selon quelles modalités? Quels peuvent être les dispositifs ou mécanismes déclencheurs d'une telle dynamique ? Quelle place les acteurs peuventils y occuper ? Ces questions, nous les formulerons et nous les traiterons dans le cadre de ce qui semble se présenter aujourd'hui comme le paradigme de l'instrumentation de gestion. Destinés au recueil et à l'analyse comparative, prévisionnelle et rétrospective des informations relatives au fonctionnement organisationnel, les outils de gestion doivent permettre la mise en place de l'action collective organisée, ainsi que le pilotage de la performance. Toutefois, par la problématique sociale et communicationnelle qu'ils soulèvent, ils 
dépassent largement le domaine comptable et financier, d'où ils tirent pourtant leur origine. L'idée que les outils de gestion (parmi lesquels les tableaux de bord, le reporting, la planification PERT, le ROI, les ERP...) sont porteurs de changement est largement admise, bien que des désaccords subsistent aujourd'hui encore sur les mécanismes impliqués. Nous examinerons deux points de vue. Selon la perspective gestionnaire (Moisdon, 1997; Bancel-Charansol et Jougleux, 2004), fortement teintée de fonctionnalisme, le pouvoir structurant des outils de gestion peut revêtir quatre formes principales. La première correspond à leur capacité à normer le comportement des acteurs en vue d'atteindre un optimum, la plupart du temps défini par la hiérarchie. La seconde renvoie à leur aptitude à révéler des dysfonctionnements organisationnels, réels ou perçus, et à suggérer des axes d'améliorations possibles (référentiels qualité, tableau de bord...). Par ailleurs, bien que n'intervenant pas comme catalyseur direct du changement, certains outils agissent en tant que support d'accompagnement de la mutation, envisagée et planifiée avant même l'implantation de l'outil. Celui-ci doit permettre la mise en œuvre du changement en facilitant le travail de mise en commun des représentations. Précisons que l'élaboration de la vision partagée ne concerne pas la totalité de l'organisation : elle se cantonne aux acteurs qui appartiennent à un même secteur et utilisent le même outil. Enfin, par le capital de connaissances nouvelles qu'il introduit, l'outil de gestion peut conduire à une mise en cause des savoirs techniques existants. Révélant l'insuffisance des pratiques et des méthodes antérieures, il suscite le besoin de développer des modes opératoires plus adaptés. Aussi est-il à même de déclencher un processus d'apprentissage organisationnel.

\section{Vers une approche pragmatique de l'impact de l'instrumentation de gestion}

L'approche gestionnaire a le mérite de mettre au jour la diversité des mécanismes de changement, mais l'inconvénient d'évacuer la part sociale et non quantifiable de l'outil. Elle tend à reléguer à l'arrièreplan les logiques de l'acteur, passant sous silence le fait que la conception, la mise en place et l'utilisation de l'instrument restent engluées dans des relations de pouvoir. Les changements organisationnels observés ne découlent pas des seules caractéristiques techniques de l'outil, ils dépendent aussi de ses modalités d'insertion dans les rapports sociaux qui fondent la vie organisationnelle (Lorino, 2007). Tout autrement se présente la position - résolument pragmatique de Weick. Récusant la conception de l'outil comme système de modélisation du réel, érigé en guide objectif pour l'action et la décision, 
Dossier : Conduire le changement organisationnel?

l'auteur invite à le (re)considérer sous un angle psychologique et comme un acte de langage. En tant qu'objet d'investissement affectif et foyer de cristallisation des imaginaires, l'outil de gestion a des répercussions sur la motivation des acteurs, facilitant ainsi ou entravant l'action collective. En tant que grille de lecture et de décodage de la « réalité » organisationnelle (informations comptables, comportements, prévisions), qui donne à voir certains aspects de l'organisation (et qui en occulte d'autres), il agit doublement. En premier lieu, il diffuse auprès de ses utilisateurs un nouveau langage et par conséquent, de nouvelles façons de penser l'organisation. En second lieu, et en particulier quand il s'inscrit dans une logique de domination et d'influence (et de résistance), il oriente le contenu et les modalités de l'interaction. C'est dans ce contexte que s'inscrit notre investigation. En réaction à l'approche trop étroitement gestionnaire de l'outil de gestion, en accord avec l'optique psychosociologique et pragmatique de Weick, nous admettrons que le pouvoir structurant de l'instrument dépend moins de son architecture interne que des conditions de sa réception auprès de ses utilisateurs (perception, attitude, valeurs locales...). Nous nous proposons d'étudier comment, en fonction du mode d'appropriation de l'outil, les acteurs développent à son égard une attitude positive ou négative, laquelle à son tour peut orienter les communications vers un modèle narratif ou argumentatif. Plus précisément, on s'attend à ce que dans le cas où l'introduction de l'outil résulte d'une démarche «top-down», les utilisateurs, ayant été écartés de la fixation des objectifs et des plans d'action, assignent à l'outil des significations négativement connotées. Il devrait s'en suivre une réaction de pesanteur communicationnelle, caractéristique de la rationalité argumentative (repli derrière les règles organisationnelles impersonnelles, développement des échanges formels, accentuation des communications hiérarchiques aux dépends des coordinations transversales, tendance à la justification des actes...). Dans le cas contraire d'une démarche «bottom up», qui suppose un style de direction non dirigiste et une organisation faiblement hiérarchisée, on devrait assister au déploiement d'une rationalité narrative. Perçue comme une reconnaissance de l'autre et une incitation à l'expression de soi, cette approche devrait favoriser l'expression de regards croisés sur les différents aspects de la réalité professionnelle. Les discours qui accompagnent la mise en place et l'utilisation de l'outil mêlent le «je » et le «nous», les éléments techniques autant que les vécus des situations de travail nouvelles. 


\section{Présentation de notre investigation empirique et de la méthode retenue}

Le terrain. Nous nous sommes centrés sur le changement organisationnel induit par la mise place de la démarche qualité. Celleci constitue une forme de management qui institue un mode de fonctionnement organisationnel axé sur la satisfaction de l'usager (ou du client) et la qualité des interactions entre ce dernier et les membres de l'organisation. Aux communications verticales et hiérarchiques entre acteurs, elle tend à substituer un modèle d'échanges horizontaux, supposés plus fluides (Pène, 2001; Laurens, 2003). Nous avons sélectionné deux organisations productrices de services à la personne, appartenant au secteur social et médico-social et fortement axées donc sur des activités relationnelles. L'outil de gestion retenu est le référentiel qualité qu'on peut définir comme un document comportant un ensemble d'exigences (règles de conduites, dispositions et modes opératoires) auxquelles l'établissement se doit de répondre en vue de satisfaire les attentes de l'usager (et des instances de certification...). En de nombreux points (taille, public accueilli, statut juridique, ancienneté...) les deux organisations sont comparables. Mais l'une se caractérise par la co-construction du référentiel, impliquant la participation de toutes les catégories du personnel (démarche «bottom-up»); et l'autre, par l'importation par la direction d'un outil prêt à l'emploi, élaboré par un cabinet externe (démarche «op-down»).

Démarche méthodologique ${ }^{l}$. Notre démarche s'appuie sur deux méthodes : la méthode archivistique et l'enquête par entretiens. La première nous a conduit à analyser les documents institutionnels exprimant le discours de l'organisation sur son propre fonctionnement interne, sur sa structure et sur les règles régissant les échanges entre services et entre salariés. L'objectif était de procéder à une évaluation du degré de formalisme visé par l'organisation. Il s'agit pour l'essentiel des référentiels, des comptes-rendus de réunion, du livret d'accueil et du contrat de séjour. L'examen des référentiels devait nous renseigner plus précisément sur les axes de fonctionnement considérés comme prioritaires ainsi que sur les réponses apportées par l'organisation aux situations de non-qualité. D'autre part, les entretiens non directifs devaient nous permettre de mieux cerner les modalités d'appropriation de la démarche qualité par les acteurs

\footnotetext{
${ }^{1}$ Pour plus de précisions, nous renvoyons le lecteur à la présentation originale (Aïssani, 2007).
} 


\section{Dossier : Conduire le changement organisationnel?}

concernés. Afin de pouvoir mettre en évidence les effets de la mise en œuvre de la politique qualité, nous avons programmé les entretiens neuf mois après l'introduction du référentiel. Les réponses ont été soumises à une analyse de contenu.

\section{Résultats et conclusion}

Les salariés (condition «top-down») évoquent spontanément les réunions, aussi importantes que nombreuses, qui ont ponctué la démarche. Lieu d'informations et de débats, elles ont permis à chaque intervenant d'interpeller les pratiques des collègues, de questionner le fonctionnement de l'établissement, de faire part de ses préoccupations personnelles ou d'équipe et d'interroger les résidents. Nombre de ces réunions nous sont apparues comme des espaces de mise en scène collective d'évènements passés ; les participants ont pu témoigner de leurs expériences, restituer sous forme de récits des évènements significatifs au plus près de la réalité vécue. Les séances consacrées au repérage des domaines à inclure dans le référentiel (par exemple : rapports à la famille de l'usager, procédures d'admission, vie sociale, partenariat et réseaux...) et à la définition des indicateurs ont été particulièrement éprouvantes. Elles ont en effet impliqué d'importants efforts d'imagination et de réflexion sur sa propre activité. Mais surtout, elles ont parfois donné lieu à des échanges conflictuels, à des stratégies de négociation, chaque catégorie professionnelle craignant une sous-représentation dans le référentiel. La dynamique communicationnelle et sociocognitive enclenchée par les réunions qualité a été relayée par d'autres dispositifs institutionnels (boîte à idées, enquête par questionnaire auprès des résidents). Fait intéressant, elle s'est déplacée vers d'autres espaces de communication - les échanges informels de tous les instants, les discussions de couloir.

Nous avons procédé à l'analyse de plusieurs types de documents (comptes rendus de réunion, livret d'accueil, contrat de séjour, cahier de liaison) afin d'étudier le type de réponse apportée par l'établissement aux situations de non qualité. Il peut s'agir, là, par exemple de fautes, de manquements aux règles en vigueur avec des degrés divers de gravité, imputés par le professionnel à l'usager ou l'inverse. La comparaison entre les deux structures révèle des particularités de fonctionnement particulièrement intéressantes. L'établissement qui a mis au point le référentiel qualité semble ne pas avoir prévu de procédure organisée et systématique. Si le débat contradictoire est prévu, les situations conflictuelles sont traitées «sur le tas » ou dans le bureau du directeur, sur le mode de l'explication et de la négociation. On peut voir là une marque de la culture orale, 
caractéristique du secteur médico-social. Dans l'autre établissement en revanche, la réponse aux situations litigieuses se fait dans les règles et selon la procédure hiérarchique. La position légaliste de l'institution et de la direction s'exprime nettement dans le souci d'expliciter par écrit les voies de recours possibles, les sanctions encourues...

La comparaison des deux référentiels met au jour des orientations contrastées qui vont dans le sens des résultats précédents. Le référentiel «importé » se singularise par l'accent mis sur les questions relatives au management de l'établissement, à son fonctionnement formel, à l'organisation. Celui qui a été élaboré collectivement associe les thèmes de la communication, du bien-être et de la socialisation des résidents. L'utilisation répétée de ces référentiels dans le cadre de la politique d'amélioration continue de la qualité va amener les acteurs à se décentrer des activités non valorisées, voire ignorées, par le référentiel. Les travailleurs sociaux tendront à développer des pratiques orientées vers la relation à l'usager (souci d'une qualité de la relation de service) ou vers le bon fonctionnement de la structure (souci d'une qualité des processus organisationnels).

L'ensemble des résultats obtenus suggère avec force l'existence de deux logiques de fonctionnement. D'une part, on voit émerger et se développer sous l'effet d'une co-construction d'un référentiel une organisation narrative. On voit aussi le rôle essentiel que les réunions jouent dans cette formation. On rejoint en cela la position de Weick qui, comme le rappelle Giordano (2006), «encourage [...] les réunions, [elles] constituent une miniature de l'organisation; elles sont les briques qui forment la réalité organisationnelle, en quelque sorte un laboratoire de l'élaboration du sens» (p. 164). Cette pratique, qui pourrait être généralisée à l'ensemble des outils de gestion, permettrait de limiter les dysfonctionnements organisationnels et humains provoqués par l'irruption brutale d'une instrumentation de gestion étrangère à la culture locale. D'autre part, et inversement, l'adoption unilatérale de l'outil, peu soucieuse d'accompagner la démarche par une politique de communication, tend à se solder par une perversion du dispositif : le référentiel devient un but en soi, le respect des règles, un phénomène résiduel qui vient parasiter la relation à l'usager. La rationalité argumentative qui prend place progressivement peut être interprétée comme un phénomène organisationnel réactionnel, voire comme une forme de résistance à l'ordre gestionnaire, et à ses piliers que sont l'évaluation, le contrôle et la quantification. Nous pensons que la distinction entre l'organisation narrative et l'organisation argumentative ne saurait se réduire à une opposition entre deux «styles» de communication 
Dossier : Conduire le changement organisationnel?

organisationnelle. Le passage de l'une à l'autre gagnerait à être pensé au travers de la problématique du pouvoir.

\section{Bibliographie}

AISSANI, Youcef. Référentiel qualité et structure de communication. Document manuscrit, 2007, p. 1-22.

AISSANI, Youcef, BORDES Odile. Démarche qualité, sensemaking et émergence dans des structures de communication. Revue Internationale de Psychosociologie, 2007, n 29, p. 59-90.

ALTER, Norbert. L'innovation ordinaire. Paris : Presses Universitaires de France, 2000, 278 p.

BANCEL-CHARENSOL, Laurence, JOUGLEUX, Muriel. L'impact des outils de gestion sur le travail en «front office». Travail et Emploi, 2004, n99, p. 103-119.

BURNS, Tom, STALKER, Georges M. The management of innovation. London : Tavistock, 1961.

LAURENS, Patrick. L'impensable communication dans les normes de système de management de la qualité. Communication et Organisation, 2003, n 23 , p. 135-150.

GIORDANO, Yvonne. S'organiser c'est communiquer : le rôle fondateur de la communication dans l'organizing chez Karl E. Weick. In : DAUTISSIER, J.F. et BENSEBAA, F. Coord. Les défis $d u$ sensemaking en entreprise. Paris : Economica, 2006, p. 153-168.

LORINO, Philippe. Un défi pour les sciences de gestion : le tournant paradigmatique du modèle de la décision au modèle de l'activité collective. In : MARTINET, A.C. Coord. Sciences du management : épistémique, pragmatique et éthique. Paris : Vuibert, 2007, p. 69-84.

MOISDON, Jean-Claude, coord. Du mode d'existence des outils de gestion. Paris : Seli Arslan. 1997. 286 p.

PENE, Sophie. Les agencements langagiers de la Qualité. In : BORZEIX, A. et FRAENKEL, B. Coord. Langage et Travail : communication, cognition et action. Paris : CNRS Editions, 2001, p. 303-322.

TAYLOR, James R., The communicational basis of organization, between the conversation and the text. In : PUTMAN, L.L et KRONE, K.K. coord., Handbook of organizationnal communication. London : Sage, 2006. 
De l'organisation narrative à l'organisation argumentative

WEICK, Karl E. Making sense of the organization. Oxford: Maiden Blackwell. 2001, 323 p.

WEICK, Karl E., BROWNING Larry D., Argument and narration in organizational communication. Journal of Management, 1986, $\mathrm{n}^{\circ} 12$, p. 243-259. 
